\title{
Average Energy Efficiency Contours with Multiple Decoding Policies
}

\author{
A. Akbari, Student Member, IEEE, M. A. Imran, R. Hoshyar, Member, IEEE, A. Amich, Student Member, IEEE, \\ and R. Tafazolli, Member, IEEE
}

\begin{abstract}
This letter addresses energy-efficient design in multiuser, single-carrier uplink channels by employing multiple decoding policies. The comparison metric used in this study is based on average energy efficiency contours, where an optimal rate vector is obtained based on four system targets: Maximum energy efficiency, a trade-off between maximum energy efficiency and rate fairness, achieving energy efficiency target with maximum sum-rate and achieving energy efficiency target with fairness. The transmit power function is approximated using Taylor series expansion, with simulation results demonstrating the achievability of the optimal rate vector, and negligible performance difference in employing this approximation.
\end{abstract}

Index Terms-Contour, Energy Efficiency, Multiple Access Channel, Uplink.

\section{INTRODUCTION}

$\mathbf{E}$ NERGY consumption has become an increasingly important aspect of wireless communications, from both an economical and environmental point of view. Over the past decade, global warming has become an important issue, with information and communication technologies (ICT) causing around $2 \%$ of the world-wide $\mathrm{CO} 2$ emissions [1]. The challenge now, is to make sure this percentage does not grow. Much of the earlier research has taken throughput maximization as the main optimization metric, with limited work covering energy efficiency. The fundamental results of energy-efficient point to point links can be traced back to [2], [3], where the capacity in bits-per-joule is given for a single link on flat fading and frequency selective channels.

In this work, the average energy efficiency is defined as the optimization metric for a $K$-user multiple access channel (MAC), where each user has an individual transmit power constraint. Based on the assumption of using a successive interference cancellation (SIC) receiver, two decoding policies are employed. Each decoding policy is then used to calculate the transmit power and average energy efficiency for a fixed data rate. A framework is then created to choose the decoding policy that achieves the highest energy efficiency for each rate vector. Next, mathematical simplifications are introduced on the energy efficiency function by using Taylor Series (TS) expansions, and approximating the transmit power. Simulation results demonstrate negligible performance difference in employing this approximation.

The authors are with the Centre for Communication Systems Research, University of Surrey, Guildford, Surrey, GU2 7XH, UK (e-mail: a.akbari@surrey.ac.uk)

\section{SySTEM MODEL}

Consider a single-carrier uplink channel, with $K$ transmitters and a single receiver, where the received signal is corrupted by AWGN with power spectral density (PSD) $N_{0} / 2$. The channel power gain from the $k^{t h}$ mobile stations (MS) to the base station (BS) is denoted as $g_{k}$. Rather than decoding each user and treating the interference from the other users as noise, an SIC receiver is used to achieve capacity [4].

The rate of user $k$ is given by the well-known Shannon formula [5] as

$$
R_{k} \leq B \log _{2}\left(1+\frac{p_{k} g_{k}}{N_{0} B}\right), \quad k=1,2, \ldots, K
$$

where, $p_{k}$ denotes the transmit power of user $k$, and $B$ is the system bandwidth

The sum of rates for all users cannot exceed the capacity of a point-to-point AWGN channel with received power equal to the sum of received powers from all users. This is also shown in the formulation of the capacity region of a $K$-user Gaussian MAC which was shown in [6] to be

$$
\begin{aligned}
& C_{M A C}(\boldsymbol{P} ; \boldsymbol{g})= \\
& \left\{\left(R_{1}, \ldots, R_{K}\right): \sum_{k=1}^{K} R_{k} \leq B \log _{2}\left(1+\frac{\sum_{k=1}^{K} p_{k} g_{k}}{N_{0} B}\right)\right\}
\end{aligned}
$$

where, $\boldsymbol{P}=\left[p_{1}, p_{2}, \ldots, p_{K}\right]$ and $\boldsymbol{g}=\left[g_{1}, g_{2}, \ldots, g_{K}\right]$ represent the power constraint and channel gain vectors respectively.

\section{ENERGY EFFICIENT DESIGN}

This section investigates the bits-per-joule measure of the system by obtaining the average energy efficiency contours using two decoding orders and finding the maximum achievable energy-efficient rate vector.

Energy efficiency is defined as the number of bits transmitted per Joule of energy. In addition to the transmit power, some power is consumed in the circuitry or dissipated in the form of heat, which is defined as the circuit power $\left(P_{c}\right)$. Throughout this work, it is assumed that $P_{c}$ is a fixed value, independent of the transmission state and equal for all users.

The overall energy efficiency of user $k$ is defined as

$$
E E_{k}=\frac{R_{k}}{P_{c}+p_{k}}, \quad k=1,2, \ldots, K
$$

The comparison metric used in this study is based on the average energy efficiency of the system defined as

$$
E E_{A V}=\frac{1}{K} \sum_{k=1}^{K} E E_{k}
$$


where, each $E E_{A V}$ value corresponds to an energy efficiency contour.

The decoding order will define the transmit power of each user, which will equally affect the energy efficiency contours. To discuss the decoding polices, $\pi$ is defined as the permutation of the ordered sequence, where $\pi(k)$ is the $k^{t h}$ element of the permutation.

Policy $1\left(\mathcal{P}_{1}\right)$ : Corresponds to scenarios where user $K$ is always decoded first, which means that user 1 can achieve its single user bound, with other users achieving a non-zero rate.

$$
\mathcal{P}_{1}: \quad \pi=\{K, K-1, \ldots, 1\}
$$

Policy $2\left(\mathcal{P}_{2}\right)$ : Decoding policy 2 reflects the reverse scenario to policy 1 , where user 1 is always decoded first.

$$
\mathcal{P}_{2}: \quad \pi=\{1,2, \ldots, K\}
$$

Since it is assumed that an SIC receiver is employed, the user decoded last can achieve its single-user bound whilst other users experience some finite multi-user inference based on their position in the decoding order. By employing either decoding policy, the transmission rate of user $k$ is given as

$$
R_{\pi(k)}=B \log _{2}\left(1+\frac{p_{\pi(k)} g_{\pi(k)}}{N_{0} B+\sum_{j=1}^{k-1} p_{\pi(j)} g_{\pi(j)}}\right)
$$

where, the second term in the denominator represents the accumulated interference from other users.

Conversely,

$$
p_{\pi(k)}=\frac{N_{0} B}{g_{\pi(k)}}\left(2^{\frac{R_{\pi(k)}}{B}}-1\right)\left(\prod_{j=1}^{k-1} 2^{\frac{R_{\pi(j)}}{B}}\right)
$$

Based on the choice of decoding policy, (4) is used to obtain the average energy efficiency metric, where $E E_{A V}^{\mathcal{P}_{1}}$ and $E E_{A V}^{\mathcal{P}_{2}}$ denote cases where decoding policy 1 and 2 have been chosen respectively.

The optimal energy efficiency is found such that, for each rate vector, the decoding policy that achieves the highest energy efficiency is chosen.

$$
E E_{O p t}=\max \left(E E_{A V}^{\mathcal{P}_{1}}, E E_{A V}^{\mathcal{P}_{2}}\right)
$$

Algorithm 1 summarizes the technique used for finding the maximum achievable energy efficiency.

\section{Optimality Measures}

This section presents the average energy efficiency contours of the system and locates the optimal operating point using several criteria of optimality, which are discussed in subsequent sections.

\section{A. Maximum Energy Efficiency}

This criterion corresponds to scenarios where the only aim of the system is to maximize the overall energy efficiency,
ALGORITHM 1: Finding Maximum Energy Efficiency

$$
\begin{array}{ll}
\text { for }\left(R_{1}, \ldots, R_{K}\right) & \\
\text { From } \mathcal{P}_{1} \text { and } \mathcal{P}_{2}: & \text { Caclulate corresponding }\left[p_{1}, \ldots p_{K}\right] \\
& \text { Use (3) and (4) to calculate } E E_{A V} \\
\begin{array}{l}
\text { Using (9): } \\
\text { Repeat for all rates }
\end{array} & \text { Find } E E_{O p t}=\max \left(E E_{A V}^{\mathcal{P}_{1}}, E E_{A V}^{\mathcal{P}_{2}}\right) \\
\text { end }
\end{array}
$$

without any consideration on user-fairness or sum-rate maximization.

$$
\begin{aligned}
& \max _{R_{1}, \ldots, R_{K}}\left(E E_{O p t}\right) \\
& \text { s.t. }\left(R_{1}, \ldots, R_{K}\right) \in C_{M A C}(\boldsymbol{P} ; \boldsymbol{g})
\end{aligned}
$$

where, constraint (11) specifies that all rate pairs should be chosen within the capacity region boundary. The optimal rate pair is chosen such that $E E_{O p t}$ is maximized, and is marked with a cross in Fig. 1.

\section{B. Maximum Energy Efficiency and Rate Fairness}

This criterion investigates scenarios where the aim is to consider rate fairness between users as well as achieving the maximum possible energy efficiency. Points with equal rate share for all users lie on a line passing through the origin with a slope of +1 (line of fairness). The optimization problem with the added rate-fairness constraint is shown as

$$
\begin{array}{ll}
\max _{R_{1}, \ldots, R_{K}}\left(E E_{O p t}\right) \\
\text { s.t. } & \left(R_{1}, \ldots, R_{K}\right) \in C_{M A C}(\boldsymbol{P} ; \boldsymbol{g}) \\
& R_{1}=R_{2}=\ldots=R_{K}
\end{array}
$$

Based on the chosen system parameters, the line of fairness does not pass through the optimal energy efficient point, therefore the maximum achievable energy-efficient rate vector will be the point on the energy efficiency contour tangentially touching the line of fairness, shown as point $\mathbf{B}$ in Fig. 1.

\section{Target Energy Efficiency with Maximum Sum-Rate}

For a fixed energy efficiency target $\left(E E_{T}\right)$, only one of the contours will be of interest to the system (as an example, consider contour labeled 1 in Fig. 1). The main target of this section is to maximize the sum-rate by achieving this energy efficiency target.

$$
\begin{array}{ll}
\max & \left(\sum_{i=1}^{K} R_{i}\right) \\
\text { s.t. } & \left(R_{1}, \ldots, R_{K}\right) \in C_{M A C}(\boldsymbol{P} ; \boldsymbol{g}) \\
& E E_{A V}=E E_{T}
\end{array}
$$

The point on the desired contour tangentially touching the line of constant sum-rate (slope -1) will give the rate vector achieving the target energy efficiency whilst having the maximum possible sum-rate, shown as point $\mathbf{D}$ in Fig. 1. 


\section{Target Energy Efficiency with Rate Fairness}

The intersection of the line of fairness and the desired energy efficiency target (same contour as previous section) will give the optimal rate pair for this specific criterion.

$$
\begin{array}{ll}
\max & \left(\sum_{i=1}^{K} R_{i}\right) \\
\text { s.t. } & \left(R_{1}, \ldots, R_{K}\right) \in C_{M A C}(\boldsymbol{P} ; \boldsymbol{g}) \\
& E E_{A V}=E E_{T} \text { and } R_{1}=R_{2}=\ldots=R_{K}
\end{array}
$$

It can be seen in Fig. 1 that there are two intersections (point $\mathbf{A}$ and $\mathbf{C}$ ), with both meeting the required criteria in terms of fairness and energy efficiency. However, the obvious choice in this case would be to operate at point $\mathbf{C}$, since it also has a higher maximum sum rate.

\section{Transmit Power Approximation}

For a given decoding policy, (4) provides the function which is used for plotting the energy efficiency contours. However, solving this will not give a closed form solution. To tackle this issue, the Taylor series expansion is applied in a neighborhood of zero and the transmit power function is approximated as a finite sum of $N$ terms, shown as

$$
\begin{aligned}
p_{\pi(k)}=\frac{N_{0} B}{g_{\pi(k)}}\left[\sum_{n=1}^{N} \frac{1}{n !}\left(\frac{\ln (2) R_{\pi(k)}}{B}\right)^{n}\right] \\
\\
{\left[\prod_{j=1}^{k-1} \sum_{n=0}^{N} \frac{1}{n !}\left(\frac{\ln (2) R_{\pi(j)}}{B}\right)^{n}\right] }
\end{aligned}
$$

where $N$ is the order of the Taylor series and defines the accuracy of the approximation.

\section{Simulation Results}

To simplify the exposition of concepts and ease of graphical representation, a 2-user case is considered here, but the general properties and performance measures hold for $K>2$. Consider a 2-user AWGN MAC with, $\boldsymbol{g}=[0.008,0.004]$, $\boldsymbol{P}=[300 \mathrm{~mW}, 300 \mathrm{~mW}]$ and $P_{c}=20 \mathrm{~mW}$. The system bandwidth is $B=100 \mathrm{KHz}$ and $N_{0}=10^{-9} \mathrm{~W} / \mathrm{Hz}$.

Fig. 1 presents the results for the uplink capacity region (pentagon shaped), energy efficiency contours, and optimal energy-efficient rate pair based on different optimality measures as discussed in section IV. It should be noted that any segment of the energy efficiency contours that reach outside the capacity region will not be achievable.

Fig. 2 demonstrates that for a fixed energy efficiency target $\left(E E_{T}=2.2 \mathrm{Mbits} / \mathrm{Joule}\right.$ in this example), the Taylor series approximation converges to the target contour. It is clearly shown that the accuracy of the approximation increases with $N$, and an order of 5 is sufficient for the approximation to converge to $E E_{T}$ with negligible performance difference.

\section{CONCLUSION}

In this letter, the average energy efficiency is defined as the optimization metric for a 2-user MAC, where multiple decoding policies are employed to locate the optimal rate

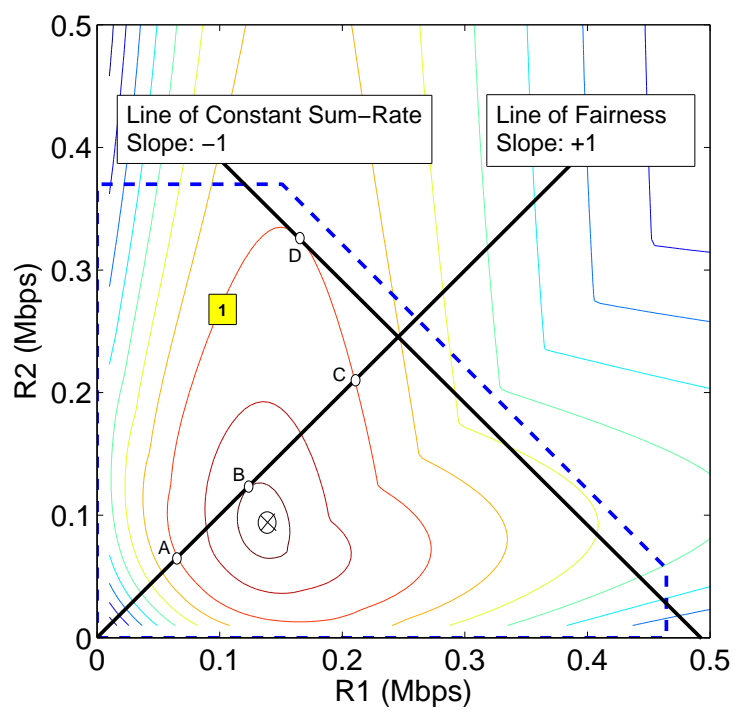

Fig. 1. Average energy efficiency contours with multiple decoding policies

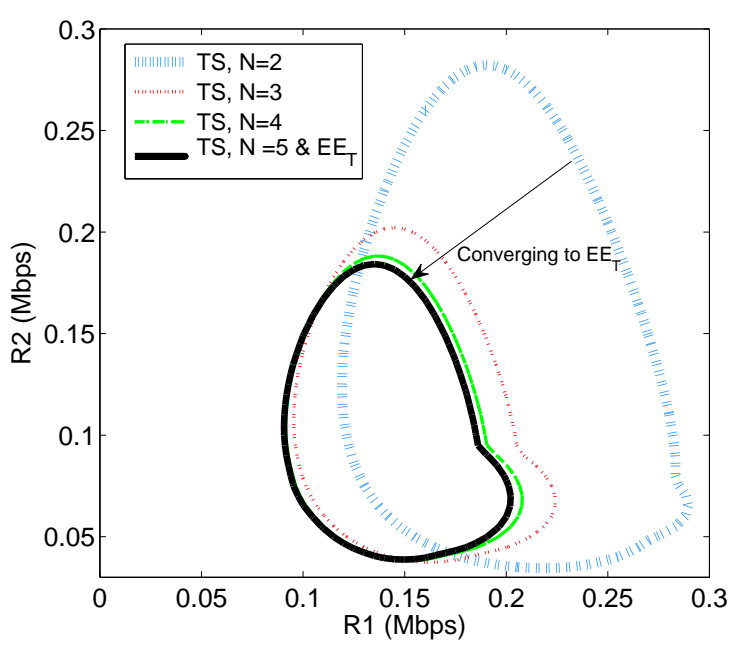

Fig. 2. Convergence of TS approximation with different accuracy orders

vector based on several optimality criteria. The transmit power function is approximated using Taylor series expansion, with simulation results verifying the achievability of the target energy efficiency with negligible performance differences using a Taylor series approximation with an order of 5.

\section{REFERENCES}

[1] S. Vadgama,"Trends in green wireless access," Fujitsu Scientific \& Technical Journal (FSTJ), vol. 45, no. 4, pp. 404-408, Oct. 2009.

[2] M. J. E. Golay, "Note on the theoretical efficiency of information reception with PPM," in Proc. IRE 1949, vol. 37, no. 1, pp.1031, Sept. 1949.

[3] H. M. Kwon, T. G. Birdsall, "Channel Capacity in Bits per Joule," IEEE Journal of Oceanic Engineering, vol. 0E-11, no. 1, pp.97-99, Jan. 1986.

[4] D. Tse and P. Viswanath, Fundamental of Wireless Communication, Cambridge University press, 2005.

[5] C. E. Shannon and W. Weaver, A Mathematical Theory of Communication. Urbana, IL: University Illinois Press, 1949.

[6] T. Cover and J. Thomas, Elements of Information Theory. New York: Wiley, 1991. 